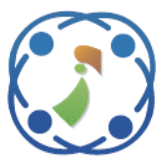

\title{
A New Robust Decoding Technique of Four Transmitters MIMO STBC System based on FastICA Algorithm
}

\author{
Kasim K. Abdalla ${ }^{1 *}$ \\ Sameer Abdul Kadhim Alrufaiaat ${ }^{1}$ \\ ${ }^{1}$ Electrical Engineering Department, Collage of Engineering, Babylon University, Babylon, Iraq \\ * Corresponding author's Email: kasimkaa.11@gmail.com
}

\begin{abstract}
A new robust decoding technique which designed of Multiple-Input Multiple-Output Space-Time Block Code (MIMO-STBC) using Fast Independent Component Analysis (Fast-ICA) based on proposed mixing model has been performed in this paper. This decoding technique is characterized by i) complexity is very low, ii) the speed is high and iii) BER performance is excellent. It can be achieved with any MIMO STBC system with a fewer pilot symbols number. Also, it is reduced decoding time into $1 / 8$ by innovating a simple strategy referred by one source extraction method. Also, this paper includes suitable initializing for the de-mixing vector to solve the ambiguities problem of sign and source of blind source separation (BSS). To test the proposed technique, four transmitters (4Tx) STBC MIMO system was implemented using MATLAB2018. It also found that excellent BER performance associated with a high number of symbols per frame (about 8012 symbols). The simulation results show that the new decoder works for any number of receiver antenna $\left(N_{r}=2,4\right.$ and 5). As compare with classical decoding algorithm, it is found that the new decoder provides coding gain (at BER $=10^{-6}$ ) equal to $1 \mathrm{~dB}, 1.45 \mathrm{~dB}$ and $1.76 \mathrm{~dB}$ when $N_{r}=2,4$ and 8 respectively, using only 2 3 iterations only.
\end{abstract}

Keywords: Space-time block coding (STBC), Transmit diversity, Multiple input multiple output (MIMO).

\section{Introduction}

Communication systems have become essential in our daily life and cannot be dispensed with. Therefore, in recent years, high data rates have been increased dramatically for the demand wireless communication system. In modem communication systems, Multiple-Input Multiple-Output (MIMO) is a one from the extreme significant technical to improve communications reliability by combating multipath fading, where the taramite signal that processed of time is complemented with spatial dimension by distributing multiple antennas spatially at both transmit and receive ends [1].

STBCs are an emerged version from Alamouti method [2, 3], but have the same key specifications. The STBCs are example of orthogonal codes which can perform full transmit diversity that affected by the transmit antennas number. In addition, STBCs can be considered as a complex version, that means, STBCs schemes of the encoding and decoding on both the transmitter and receiver sides are the same as there in the Alamouti space-time code. STBCs were introduced to perform the maximum order of the diversity for the same transmit and receive antennas number, subjecting to the constraint to have a decoding algorithm with simple linear feature, hence, STBCs became a very common scheme and most widely achieved in communication system [4].

When the transmit signals burst through the channel, processing of channel estimation has been utilized to provide essential information about the diverse interactions which are happened to the data signal, hence, the researcher interested by the design of the estimation of the channel. Channel state information (CSI) has been provided by various suggested strategies and achieved for wireless channel estimation. The MIMO type in wireless communication technology at the receiver end, effective developed strategies have been promoted newly for channel estimation. 
In MIMO transmission, there are three groups of estimation strategies can be divided in [3]:

Non-Blind Channel Estimation: This technique, pilot symbols and Channel State Information (CSI) are used to estimating the channel response. The disadvantage of using pilot symbols is the reduction of transmission efficiency.

Blind Channel Estimation: This technique does not require pilot symbols. It provides full transmission efficiency.

Semi-Blind of Channel Estimation: This technique requires fewer number of pilots that reduces transmission efficiency.

In this paper, FastICA algorithm where used as BSS technique to the build a new proposed decoder. This technique supposes that there is a single value for the de-mixing vector that maximizes Negative Entropy measuring in any mixing system. This work proposed a new a new model for MIMO STBC based on representing maximum ratio combiner as linear mixing system. This new mixing model gives us opportunity to decode the MIMO STBC even though number of receivers less than number of transmitters. It's also denoted that the new mixing matrix is always orthogonal that eliminating the needs for pre whiting step and one-unit FICA could be applied efficiently. A suitable initializing for de-mixing vector innovated to get rid of sign and source ambiguities problem of associated with Fast ICA algorithm. The robust points in this new decoder that it's no need channel estimator. In other words, our proposed strategy can be used for estimation and decoding at same time. The second robust point that decoding time reduced by $1 / 8$ using a simple strategy referred by one source extraction method.

The main goal of this paper is to provide STBCs for four transmit antennas and multiple receive antennas using semi-blind of channel estimation, improve BER performance, reduce decoding time and solve the ambiguities of BSS.

The paper outline is presented as follows. A mathematical model for four transmitters MIMOSTBC system has been presented in Section 2, denoted that the performance of MIMO STBC counts essentially on the channel estimator quality. Section 3 reviews channel estimator and Least Square (LS) technique for MIMO channel, stating multiple transmitter antennas block diagram model. Section 4 states Blind Source Separation (BSS) technique, emphasizing on the nonquadratic function and the popular ones suggested by Hyvärinen, shows a block diagram of one-unit FastICA algorithm and presents proposed mathematical model, denoting that mixing matrix is always orthogonal and one-unit FICA could be applied efficiently. The proposed decoder and the algorithm steps are stated in Section 5. Section 6 shows, firstly, simulation results and the performance comparison in terms of BER of MRC decoder of MIMO STBC, then simulation and statistical analysis in term of Probability Distribution Function (PDF) of source, mixture and extraxted source signals and in term of a non-quadratic function used with FICA algorithim and its derivative. Finally, Section 7 explains some conclusions of this paper.

\section{MIMO STBC system model}

Let consider a quasi-static flat fading channel with $N_{t}$ transmitters and $N_{r}$ receiver, then received signal vector $Y^{t}=\left[\begin{array}{c}y_{1}^{t} \\ : \\ y_{N_{r}}^{t}\end{array}\right]$ at time $t$ is given by $[5,6]$ :

$$
Y^{t}=H X^{t}+\text { noise }
$$

where $X^{t}=\left[\begin{array}{c}x_{1}^{t} \\ \vdots \\ x_{N_{t}}^{t}\end{array}\right]$ is the transmitted signal vector at time $t$ and $H$ is $N_{r} \times N_{t}$ complex number channel coefficients matrix. For the quasi-static assumption, the channel reaction is changing at random between adjacent blocks, however it is not variable within time of transmission, this time is known as coherence time [5].

In this paper, four transmitters $N_{t}=4$ have been used as MIMO STBC and whose encoding matrix is define as [6]:

$$
G=\left(\begin{array}{rrrr}
S_{1} & S_{2} & S_{3} & S_{4} \\
-S_{2} & S_{1} & -S_{4} & S_{3} \\
-S_{3} & S_{4} & S_{1} & -S_{2} \\
-S_{4} & -S_{3} & S_{2} & S_{1} \\
S_{1}^{*} & S_{2}^{*} & S_{3}^{*} & S_{4}^{*} \\
-S_{2}^{*} & S_{1}^{*} & -S_{4}^{*} & S_{3}^{*} \\
-S_{3}^{*} & S_{4}^{*} & S_{1}^{*} & -S_{2}^{*} \\
-S_{4}^{*} & -S_{3}^{*} & S_{2}^{*} & S_{1}^{*}
\end{array}\right)
$$

where $S_{1}, S_{2}, S_{3}, S_{4}$ are input modulated signal of STBC encoder and the sequences of any two antennas (columns of $G$ ) of the transmitters are orthogonal [58].

In order to decode MIMO STBC system, channel coefficients must be estimated then maximum ratio combiner (MRC) decoder estimates original signals $S_{1}, S_{2}, S_{3}, S_{4}$ by combining received signal vectors $Y^{t}$ at times $t=1,2, \ldots, 8$ using the linear transformation $[7,8]$ : 


$$
\left[\begin{array}{c}
Y^{1} \\
Y^{2} \\
Y^{3} \\
Y^{4} \\
\left(Y^{5}\right)^{*} \\
\left(Y^{6}\right)^{*} \\
\left(Y^{7}\right)^{*} \\
\left(Y^{8}\right)^{*}
\end{array}\right]=\left[\begin{array}{rrrr}
\hbar_{1} & \hbar_{2} & \hbar_{3} & \hbar_{4} \\
\hbar_{2} & -\hbar_{1} & \hbar_{4} & -\hbar_{3} \\
\hbar_{3} & -\hbar_{4} & -\hbar_{1} & \hbar_{2} \\
\hbar_{4} & \hbar_{3} & -\hbar_{2} & -\hbar_{1} \\
\hbar_{1}^{*} & \hbar_{2}^{*} & \hbar_{3}^{*} & \hbar_{4}^{*} \\
\hbar_{2}^{*} & -\hbar_{1}^{*} & \hbar_{4}^{*} & -\hbar_{3}^{*} \\
\hbar_{3}^{*} & -\hbar_{4}^{*} & -\hbar_{1}^{*} & \hbar_{2}^{*} \\
\hbar_{4}^{*} & \hbar_{3}^{*} & -\hbar_{2}^{*} & -\hbar_{1}^{*}
\end{array}\right]\left[\begin{array}{l}
S_{1} \\
S_{2} \\
S_{3} \\
S_{4}
\end{array}\right]+\text { noise }
$$

where $\hbar_{i}$ is the $i_{t h}$ column of channel coefficient matrix $\mathrm{H}$.

In general, the MRC equation for orthogonal 4Tx STBC can be written as $[7,8]$ :

$$
Y_{M R C}=H_{M R C} \times\left[\begin{array}{c}
S_{1} \\
\vdots \\
S_{4}
\end{array}\right]+\text { noise }
$$

From the Eq. (4), it is seen that $\mathrm{H}_{\mathrm{MRC}}$ is an orthogonal matrix that makes the input data symbols of space time block code can be decoded simply by [8]:

$$
\left[\begin{array}{c}
\check{S}_{1} \\
\vdots \\
\check{S}_{4}
\end{array}\right] \cong \frac{1}{\left\|\mathrm{H}_{\mathrm{MRC}}\right\|}\left(H_{M R C}\right)^{H} Y_{M R C}
$$

From this equation can be denoted that the performance of multiple in multiple out STBC counts essentially on the channel estimator quality.

\section{Least square estimator for MIMO channel}

In any wireless communication system, the processing of the channel estimation is considered as a task of major challenge. The duty of estimation of channel gives enough data of distortion delays, attenuations, interferences, and phase shifts happened to the transmitted channel signals [9].

The common technique of the channel estimation is using pilot symbols $\left(X_{P}\right)$ which understood by the receiver side as explained in Fig. 1.

In order to keep track of the time-varying channel characteristics, the pilot symbols must be placed as frequently as the coherence time. The Least Square (LS) is one of the common techniques which is vastly achieved for channel estimation if symbols of training $\left(Y_{P}\right.$ and $\left.X_{P}\right)$ are obtainable and the matrix of the estimated channel is presented in below [10]:

$$
H_{L S}=Y_{P} X_{p}^{H}\left(\mathrm{X}_{\mathrm{P}} X_{p}^{H}\right)^{-1}
$$

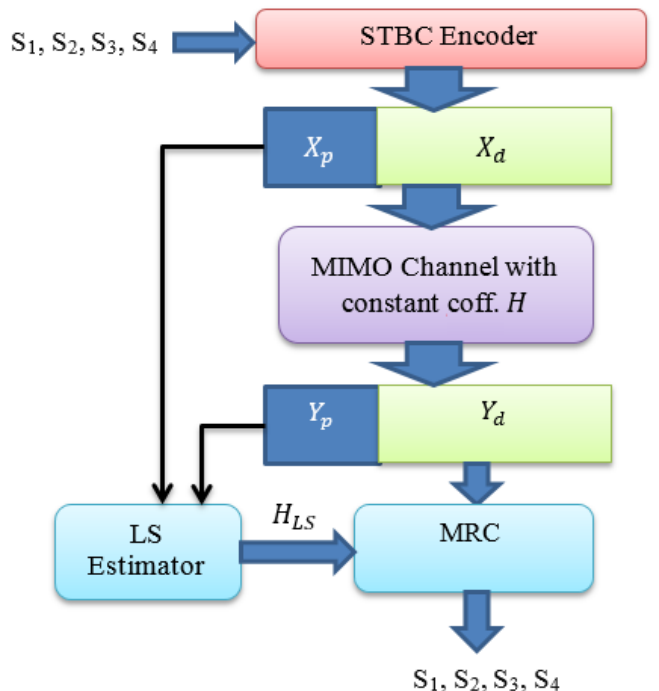

Figure. 1 Channel estimation using pilot sequence

\section{Blind source separation (BSS) technique}

BSS is an improved signal processing technique which objects to recover $n_{s}$ signals of source $U=$ $\left[\begin{array}{c}u_{1} \\ \vdots \\ u_{n_{s}}\end{array}\right]$ from a $n_{\mathrm{r}}$ received mixture signals $R=\left[\begin{array}{c}r_{1} \\ \vdots \\ r_{n_{r}}\end{array}\right]$ without any information about the independent sources. If mixing matrix $M$ is an orthogonal then one-unit version of blind source separation (extracting one source) can be accomplished by $[12,13]$ :

$$
\check{u}_{i}=w R
$$

where $w$ is de-mixing vector.

All BSS algorithm based on adjusting the parameters of de-mixing vector in order to minimization and/or maximization of one or more non- Gaussianty measuring to obtain the independence signal of its output. It assumes that the sources should be independent, non-Gaussian signals, and the mixture signals are an approach to Gaussian $[14,15]$.

To get a measurement of non Gaussianty which is zero to a Gaussian variable and usually nonnegative, differential entropy definition of a modified version, called negentropy will be used in this paper. Negentropy $J(u)$ of a random variable $(r . v) u$ is defining as the difference in entropy of $u$ and Gaussian distribution r.v with the same mean and variance, simpler approximation of negentropy was developed by Hyvärinen as following [16,17]:

$$
J(u)=\left(E\left\{G(u)-G\left(u_{\text {gauss }}\right)\right\}\right)^{2}
$$

where $\mathrm{G}(\mathrm{u})$ is a non-quadratic function. 
Table 1. Non-quadratic function suggested by hyvärinen

\begin{tabular}{|c|c|}
\hline$G(u)$ & $g(u)$ \\
\hline$\frac{1}{a} \log (\cosh (a u))$ & $\tanh (a u)$ \\
\hline$-\frac{1}{a} \exp \left(-\frac{b u^{2}}{2}\right)$ & $u \exp \left(-\frac{a u^{2}}{2}\right)$ \\
\hline$\frac{1}{4} a u^{4}$ & $a u^{3}$ \\
\hline
\end{tabular}

The chosen of this function depending on the probability distribution of source signal. There are several functions suggested $\mathrm{G}(\mathrm{u})$, the popular ones suggested by Hyvärinen are given in Table 1 where $g$ () denoted the derivative of the nonquadratic function $G($ ) [14-18].

\subsection{One unit fast ICA algorithm}

The FICA, invented by A. Hyvärinen, is a very popular and efficient algorithm that is used in many applications. It tries to maximize the negentropy by maximizing the non-Gaussianty of the mixture. Solution for one-unit BSS transformation $(\breve{\mathrm{u}}=w R)$ can be solved in simple form to search for the optimum vector $\mathrm{w}_{\mathrm{opt}}$ which maximizes the $\mathrm{J}(\mathrm{u})$ value. So, negentropy can be utilized as cost function [15]:

$$
w= \begin{cases}w_{\text {opt }} & \rightarrow J(w R) \approx \max \\ \text { else } & \rightarrow J(w R)<\max \end{cases}
$$

where $w$ is the quality of de mixing vector.

The FastICA algorithm is based on a fixed-point iteration scheme as shown in Fig. 2 for finding a maximum of the non Gaussianty of $w R$ as measured in Eq. (9).

First, the magnitude of $\frac{\partial J(\bar{u})}{\partial w}$ might be evaluated, then $w$ can be updated recursively using a simplified approximation for fixed-point Newton iteration as [14,16,17]:

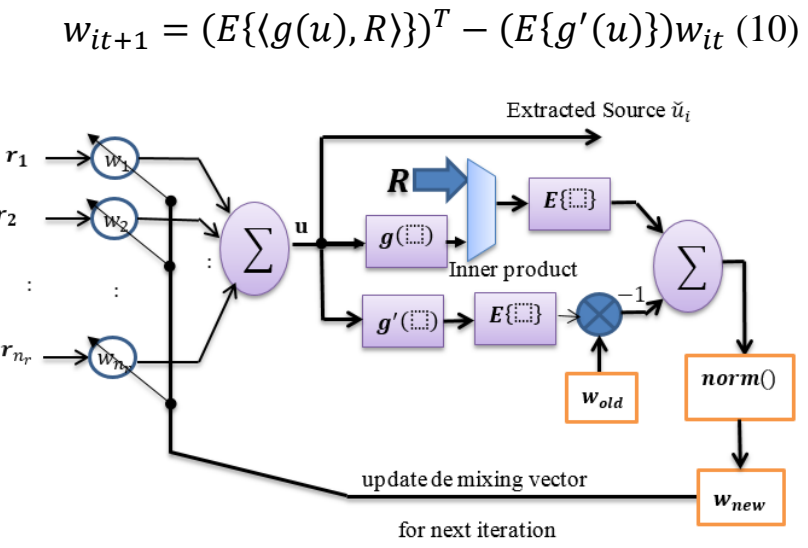

Figure. 2 One-unit fastICA algorithm where $g()$ denoted the derivative of $G()$ and $\langle$,$\rangle is$ inner product operation. Usually demixing vector must be normalized for the next iteration as shown in Fig. $2[14,18]$.

\subsection{Proposed mixing model}

In order to perform the BSS technique in decoding of four transmitter MIMO STBC system, maximum ratio complainer (MRC) decoder Eq. (4) will be modelled inform of noisy real values mixing system as following [19-23]:

$$
R=M U+\text { noise }
$$

where there are eight real values of the source signal represented in vector form:

$$
U=\left[\begin{array}{c}
\boldsymbol{u}_{\mathbf{1}} \\
\vdots \\
\boldsymbol{u}_{\mathbf{8}}
\end{array}\right]=\left[\begin{array}{c}
\operatorname{Re}\left\{\begin{array}{c}
S_{1} \\
\vdots \\
S_{4}
\end{array}\right) \\
\operatorname{Im}\left\{\begin{array}{c}
S_{1} \\
\vdots \\
S_{4}
\end{array}\right\}
\end{array}\right], n_{s}=8
$$

And the received mixture signals are arranged as:

$$
R=\left[\begin{array}{c}
\boldsymbol{r}_{\mathbf{1}} \\
\vdots \\
\boldsymbol{r}_{\boldsymbol{n}_{\boldsymbol{r}}}
\end{array}\right]=\left[\begin{array}{l}
\operatorname{Re}\left\{Y_{M R C}\right\} \\
\operatorname{Im}\left\{Y_{M R C}\right\}
\end{array}\right], n_{r}=16 N_{r}
$$

That made $n_{r} \times n_{s}$ mixing matrix $M$ is in the following form:

$$
M=\left[\begin{array}{lr}
\operatorname{Re}\left\{H_{M R C}\right\} & -\operatorname{Im}\left\{H_{M R C}\right\} \\
\operatorname{Im}\left\{H_{M R C}\right\} & \operatorname{Re}\left\{H_{M R C}\right\}
\end{array}\right]
$$

It can be denoted that mixing matrix is always orthogonal that eliminating the needs for pre whiting pre-processing step and one-unit FICA could be applied efficiently.

\section{Proposed decoder of MIMO STBC}

Due to phase and order ambiguity problems in FastICA algorithm, if the demixing vector initialized randomly (blind mode), FICA algorithm cannot be specified which source (moreover its sign) has been extracted. Therefore, demixing vector have to initialize accurately to determine which source should be extracted? [14-19].

\subsection{Initialization for FICA algorithm}

Pilot symbols are utilized to get prior knowledge for channel coefficient using LS estimator as 
described in Section 3. MRC matrix $H_{M R C}^{L S}$ can be constructed by using $H_{L S}$ then $R$-Im decomposition can be applied for $H_{M R C}^{L S}$ to obtain prior knowledge for mixing matrix $M$ which symbolize in this paper by $M_{L S}$ :

$$
M_{L S}=\left[\begin{array}{cc}
\operatorname{Re}\left\{H_{M R C}^{L S}\right\} & -\operatorname{Im}\left\{H_{M R C}^{L S}\right\} \\
\operatorname{Im}\left\{H_{M R C}^{L S}\right\} & \operatorname{Re}\left\{H_{M R C}^{L S}\right\}
\end{array}\right]
$$

In order to extract $u_{l}$ using FICA, the initial value for demixing vector and the first column of $M_{L S}$ must be equal. Also, in the same way for extracting $u_{2}$, the initial value for demixing vector and the second column of $M_{L S}$ should be equal and so on.

\subsection{The extraction of one source method}

In a sample strategy would be invited to reduce decoding delay of the proposed decoder which is applied in this paper. It's found that the proposed model of MIMO STBC gives us the opportunity to estimate the overall mixing matrix if one of its columns is known. Based on Eqs. (3) and (14), the first column of the mixing matrix $M$ appears in the following form:

$$
\begin{gathered}
\vec{m}_{1}=\left[\begin{array}{r}
k_{R} \\
k_{R} \\
k_{I} \\
-k_{I}
\end{array}\right] \text {, where, } k_{R}=\operatorname{Re}\left\{\begin{array}{l}
\hbar_{1} \\
\hbar_{2} \\
\hbar_{3} \\
\hbar_{4}
\end{array}\right\} \text { and } \\
k_{I}=\operatorname{Im}\left\{\begin{array}{c}
\hbar_{1} \\
\hbar_{2} \\
\hbar_{3} \\
\hbar_{4}
\end{array}\right\}
\end{gathered}
$$

The complex channel coefficient of MIMO channel can be found as:

$$
\left[\begin{array}{llll}
\hbar_{1} & \hbar_{2} & \hbar_{3} & \hbar_{4}
\end{array}\right]^{T}=k_{R}+j k_{I}
$$

By using the channel coefficient values, MRC matrix $\left(\mathrm{H}_{\mathrm{MRC}}\right)$ could be constructed as illustrated in Eq. (3). Finally, the whole mixing matrix could be evaluated using Eq. (14).

On the other hand, de-mixing vector or first source $w_{l}$ related to the first column of $M$ that made $w_{2}$ is related to the second column of $M$ and so on. In other words, one-unit FICA will be used only one time to estimate $w_{1}$ then the values of $w_{2}, w_{3}, \ldots, w_{8}$ could be evaluated directly as illustrated above. This strategy reduces the computing delay by $1 / 8$ times since the FastICA algorithm will apply only one time instant of applying it 8 times.

\subsection{The steps of proposed method}

The proposed decoder can be summarized in the following steps.

1. Construct $Y_{M R C}$ by combining the received signals: $Y^{1}, Y^{2}, \ldots, Y^{8}$.

2. Apply $\mathfrak{R}-\mathfrak{I} \boldsymbol{m}$ decomposition on $Y_{M R C}$ using Eq. (12) to obtain $n_{r}$ mixture signals $R$.

3. By using pilot symbols $X_{p}$ and $Y_{p}, H_{L S}$ is evaluated using Eq. (6).

4. Construct MRC matrix $H_{M R C}^{L S}$ from $H_{L S}$ then apply $\mathfrak{R}-I_{\boldsymbol{m}}$ decomposition to obtain $M_{L S}$ using Eq. (15).

5. To extract $u_{1}$ initial value for $w_{l}$ equal to first column of $M_{L S}$.

6. $i t=1$ (number of iteration)

7. Find extracted source: $u=w^{i t} R$,

8. Evaluate the functions $g(u)$ and its derivative $g^{\prime}(u)$ using Eq. (15)

9. Update the de-mixing vector using Eq. (10)

10. Normalized the weighting vector using:

$$
w^{i t+1}=\frac{w^{i t+1}}{\left\|w^{i t+1}\right\|}
$$

11. The algorithm can be stopped (go to step 12) if:

$$
\text { it }>\text { maximum number of iterations. }
$$

Or $w^{i t+1}$ converge to specific value i.e:

$|1-| w^{i t+1} \times\left(w^{i t+1}\right)^{T}|| \leq$ Threshold

else: $i t=i t+1$, Go to step 7 .

12. At end of iteration, it assumes that optimum de mixing is $w_{1}$. The values of $w_{2}, w_{3}, \ldots, w_{8}$ will be evaluate as illustrated in section 5.2. According to Eq. (15) the $i_{t h}$ complex source signal can be decoded by:

$$
S_{i}=u_{i}+j u_{i+4}=\left(w_{i}+j w_{i+4}\right) R
$$

\section{Simulation and results}

MATLAB2018b program has been used in this paper to simulate the proposed decoder of four transmitters MIMO-STBC system whose encoding matrix and MRC matrix illustrated in section 2. To test the validity of the system, a generator of random data has been used to generate information bits in the form of frame-by-frame, every frame equals $2 N_{s}$ bits 
length. QPSK modulator has been selected to perform the modulation for every frame to generate $N_{s}$ symbols. The pilot symbols, which is known to the receiver end, will be represented by the first $N_{p}$ symbols while the $N_{s}-N_{p}$ remaining symbols will represent the data symbols which have been encoded by STBC. The information encoded symbols can be transmitted by 4 antennas along with Rayleigh fading MIMO channel also a complex additive white Gaussian channel is combined with the signal of transmitter.

At the receiver side, firstly, the LS channel estimator uses pilot symbols to get the coefficient of the channel $H_{L S}$. Using the decoding algorithm described in Section 5.3, the received symbols will be decoded and send to QPSK demodulator. Eventually, the frame bits, which received from output of demodulator, will compare with originally frame bits to calculate BER with respect to a chosen signal to noise ratio. Total frames number are selected approximately $10^{4}$ frames.

\subsection{Pilot symbols number influences on conventional MRC decoder}

Fig. 3 shows the bit error rate simulation of MRC decoder for four transmitters MIMO STBC using QPSK modulator with $N_{s}=8192$ Symbols /Frame for different number of receivers and a different number of pilots. For obtaining the number of orthogonal pilot sequence, pilot symbols were set as an integer of multiple of 8 , where $N_{p}$ is chosen equal to 8,16 and 24. It's evident from the simulated figures that when pilot symbol number is increased gives significant coding for any number of receivers.

\subsection{Statistical analysis of the proposed decoder}

According to new mixing model which proposed in this paper, there are eight sources $u_{1}, \ldots, u_{8}$ and $n_{r}$ mixture signals $r_{l}, r_{2}, \ldots, r_{n r},\left(n_{r}=16 N_{r}\right)$. FICA algorithm can be applied to this model if and (only if) the two conditions of BSS are satisfied. The first condition that $n_{r}$ should be greater than $n_{s}$ for any number of receiver antennas. For the worst case if there are only two receivers $\left(N_{r}=2\right)$ that made $\left(\mathrm{n}_{\mathrm{r}}=\right.$ $32)$. Since $\left(\mathrm{n}_{\mathrm{s}}=8\right)$ that made the condition of BSS $\left(\mathrm{n}_{\mathrm{r}}\right.$ $\geq n_{s}$ ) is completely satisfied where the dimensions of mixing matrix $M$ is $\mathrm{n}_{\mathrm{r}} \times 8$.

The second condition that probability distribution of all source signals should be non-Gaussian. In this paper QPSK modulation will be used. Applying (RIm) decomposition on QPSK signal will make sources $u_{1}, \ldots, u_{8}$ as a discrete r.v which they are considered statistically of binomial distributions

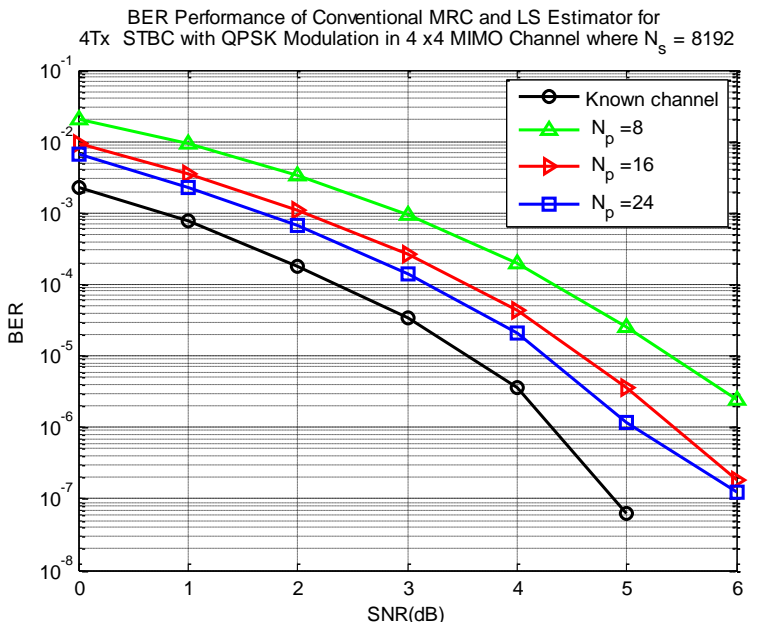

(a)

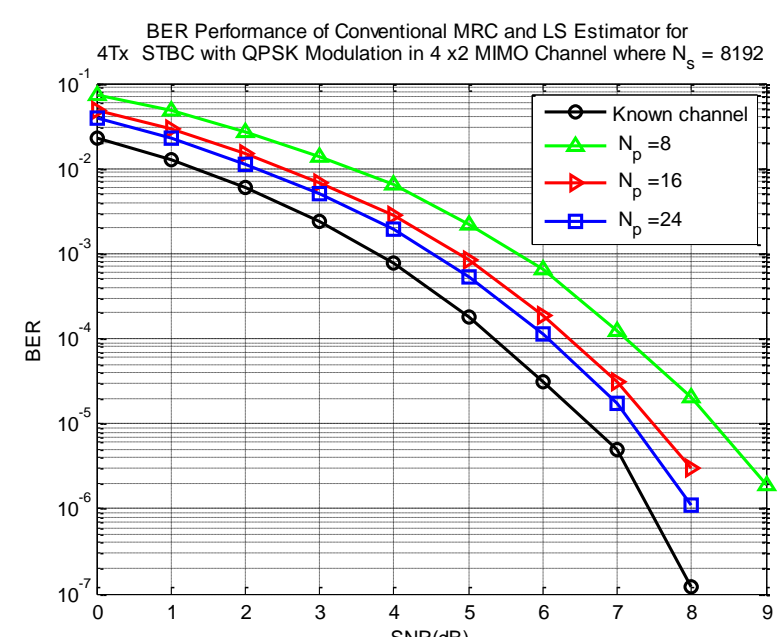

(b)

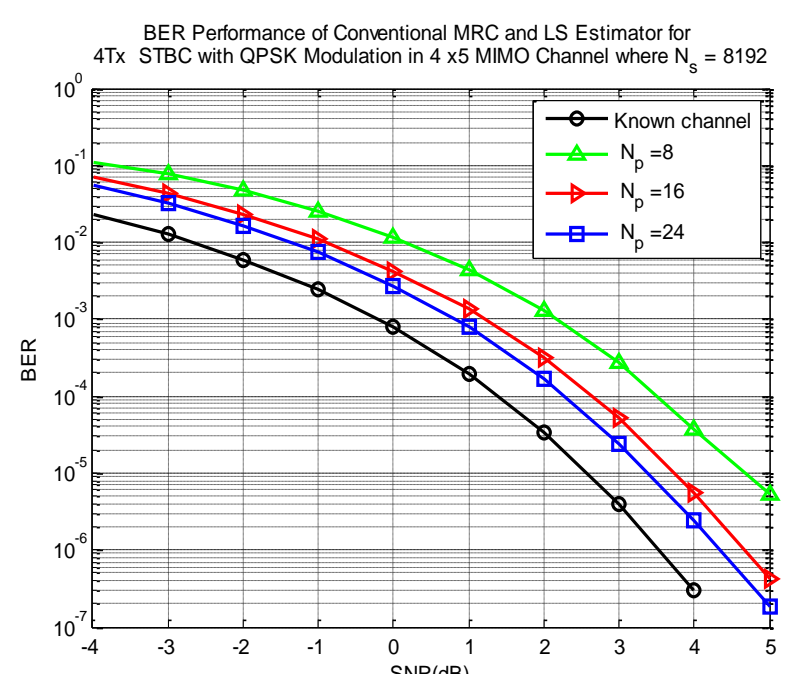

(c)

Figure. 3 BER performance of MRC decoder of MIMO STBC using a different number of pilot symbols.

represented in Fig. 4, where only one frame with 8192 symbols is used and $N_{r}=2$. If we assumed that $P_{U}(u=-1)=P_{U}(u=+1)=0.5$ that made 
the expected entropy for all source $\cong 1$ and negentropy $\cong 0.015$.

During simulation of $4 \times 2$ MIMO STBC system, it is found that the optimum quadratic function which compatible with such probability density function $(P D F)$ is given by:

$$
G(u)=\frac{1}{a} \log (\cosh (a u))
$$

That made:

$$
\begin{aligned}
g(u) & =\tanh (a u) \text { and } g^{\prime}(u) \\
& =a\left(1-\tanh ^{2}(a u)\right)
\end{aligned}
$$

where the value of $a$ is chosen equal to 1 as shown in Fig. 4.

Based on mixtures of central limit theory, $\mathrm{R}$ signals are shown as continues $r . v$ with Gaussian distribution $P D F$ as seen in Fig. 4 where $\mathrm{SNR}=8$
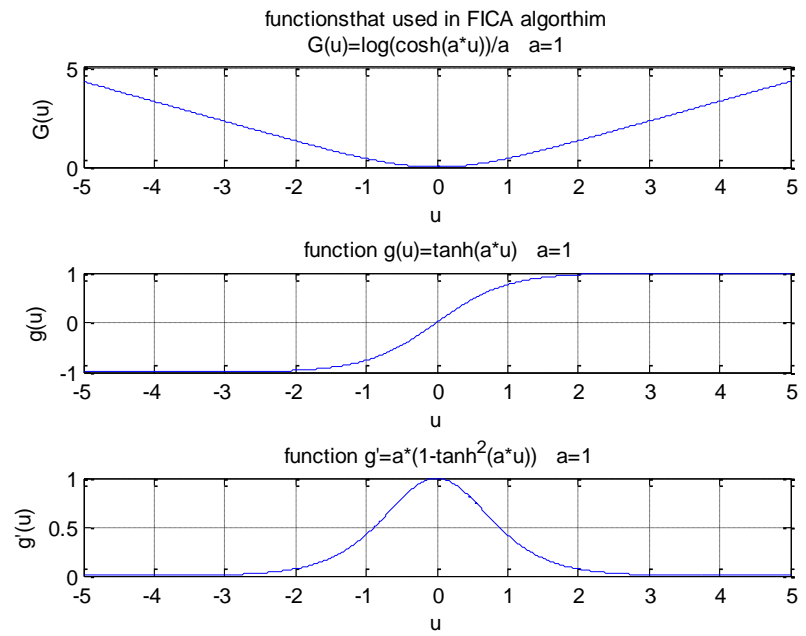

Figure. 4 Non-quadratic function used with FICA algorithim and its derivative
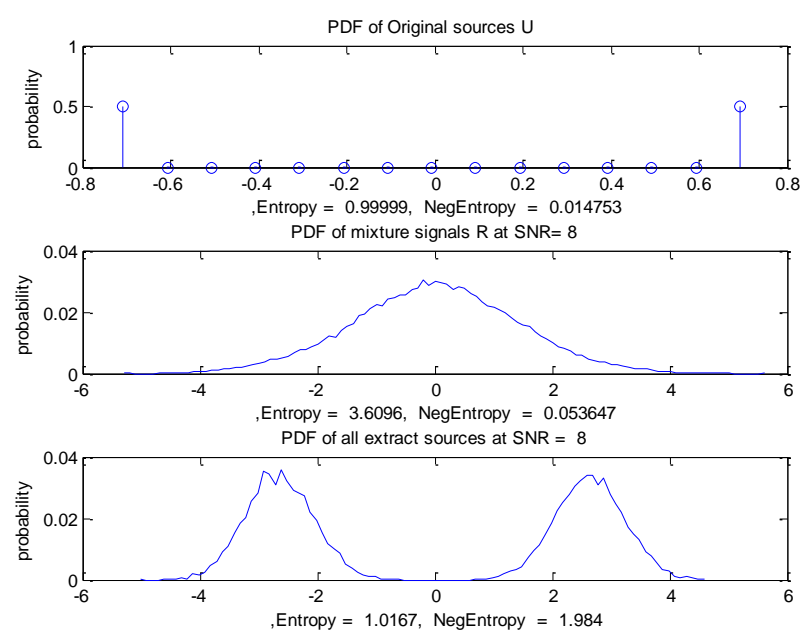

Figure. 5 Probability distribution of source, mixture and extracted source signals
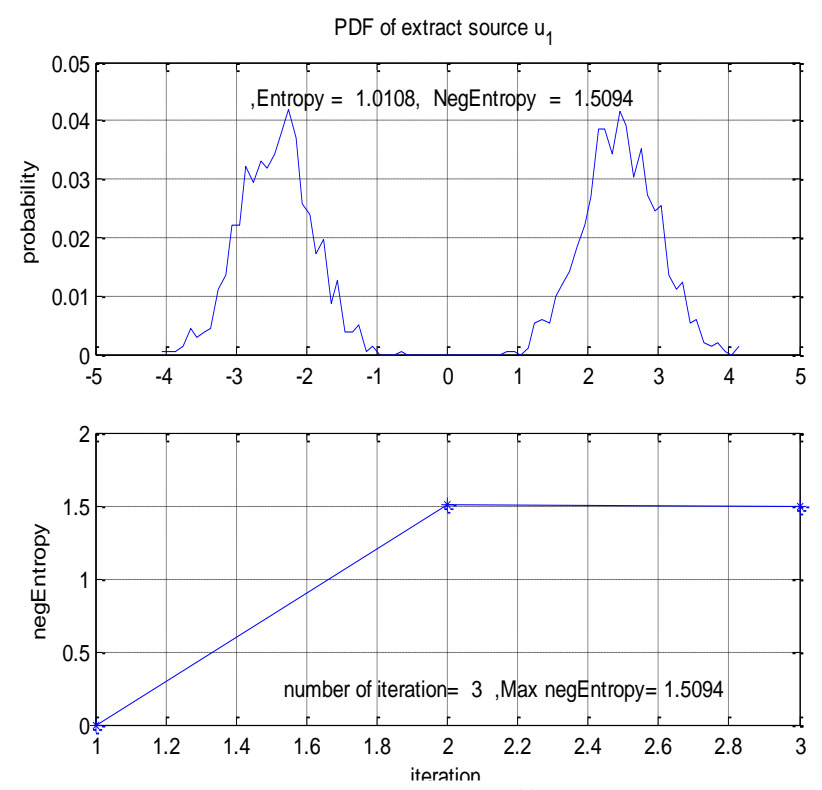

Figure. 6 Probability distribution of $\breve{u}_{1}$ and iterative update of negentropy

$\mathrm{dB}$ is used. According to this result the expected Entropy $(\mathrm{R})=3.61$ is greater than Entropy of source for any SNR value.

Now FastICA divides mixed signals, the expected $P D F$ for estimated signal $\widetilde{\boldsymbol{u}}$ is shown in Fig. 5, hence, Entropy $(\tilde{u}) \cong$ Entropy (u) at the same negentropy for all sources reach its maximum value $=1.984$.

The initial value for demixing vector is obtained from 8 pilot symbols only. One-unit FastICA used to estimate first source $u_{1}$ by update de mixing vector iteratively until negentropy reaches its maximum value $\cong 1.5094$ using only three iterations as shown in Fig. 6.

It can be denoted that the number of iterations for FastICA depends on threshold value and SNR. For checking the convergence of demixing vector into optimum position threshold has been utilized, which is specified the quality of estimation. $10^{-5}$ has been selected as threshold value in this paper. Decoder latency is reduced with decreasing threshold value, but simultaneously quality of estimation will be poor. If demixing vector doesn't converge into the specified location, then iteration is applied, 100 is assumed as the maximum number of allowed iterations.

\subsection{BER analysis of proposed decoder}

In this section we will use a minimum number of pilot symbols and test our proposed decoder with different cases:

\section{Case 1: $N_{r}=N_{t}$}

Fig. 7 shows the number of iterations and bit error rate in $4 \times 4$ MIMO using 8 pilot symbols for the 
proposed decoder. From the proposed decoder results, it is obvious that faster and excellent BER performance (approach to known channel) associated with a higher number of symbols per frame.

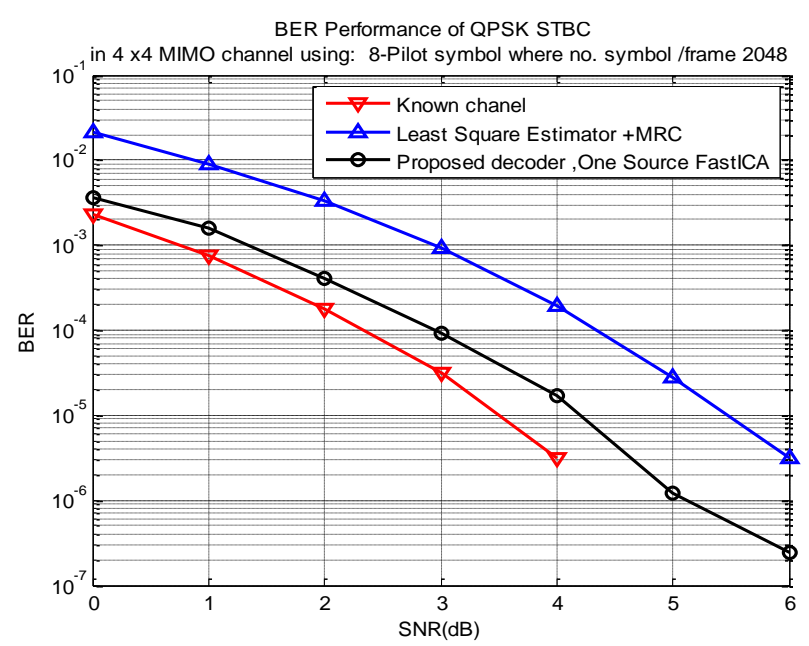

(a)

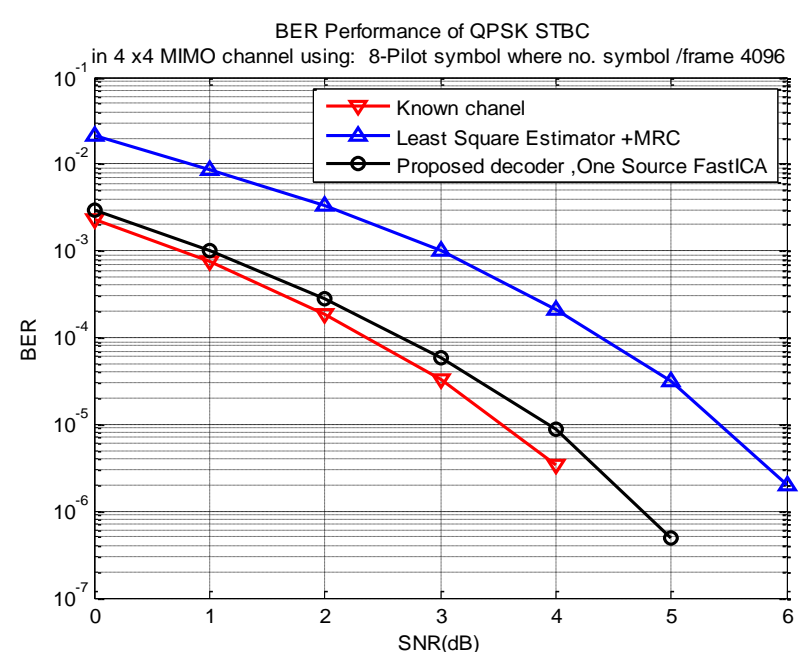

(b)

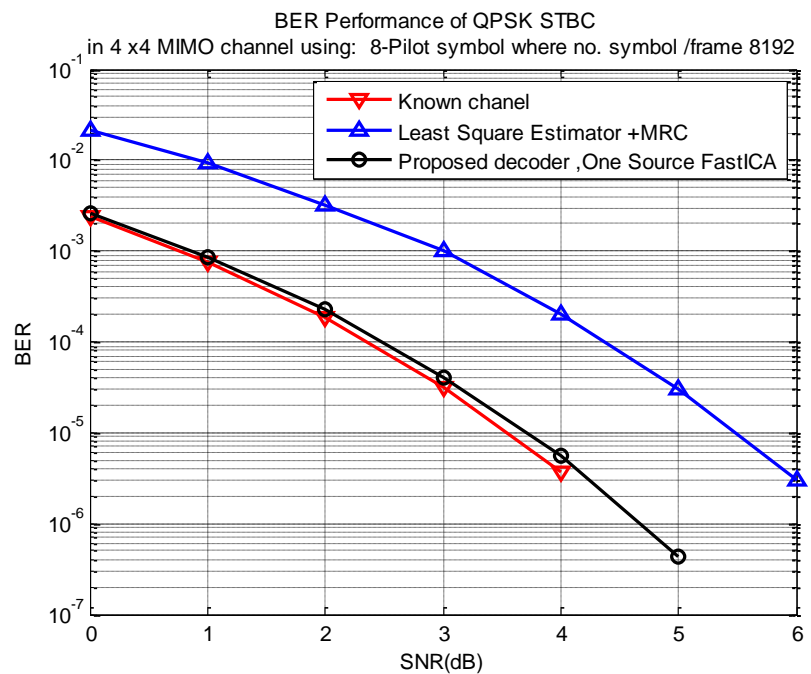

(c)

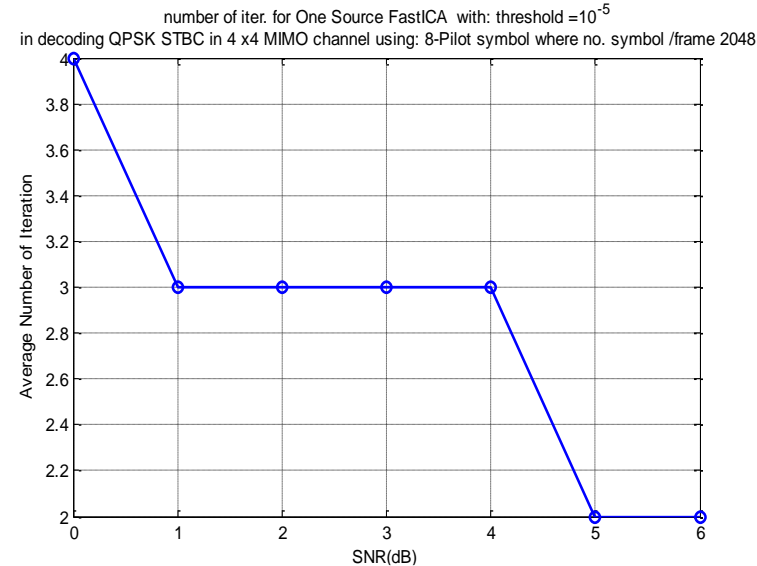

(a’)

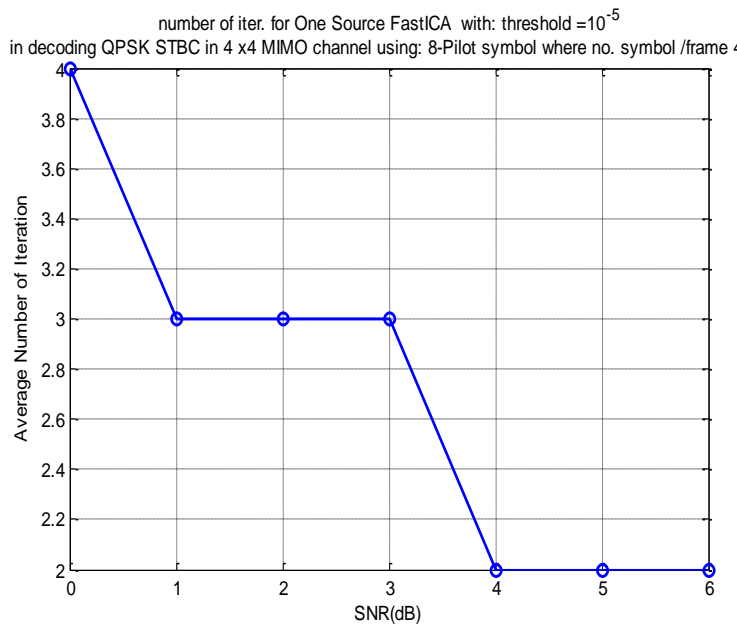

(b')

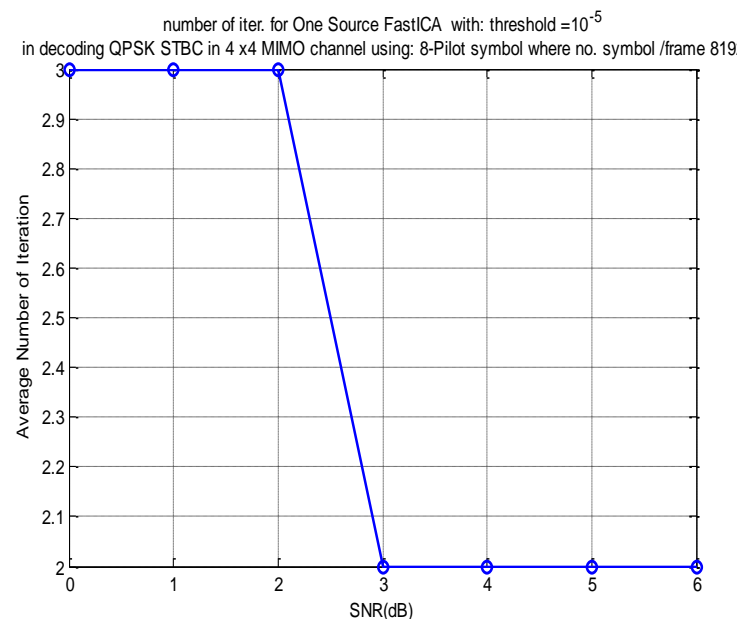

(c')

Figure. 7 Bit error rate and number of iterations for $4 \times 4$ MIMO STBC using :(a) and (a') $N_{s}=2048$, (b) and (b') $N_{s}=4096$, and (c) and (c') $N_{s}=8192$, respectively 


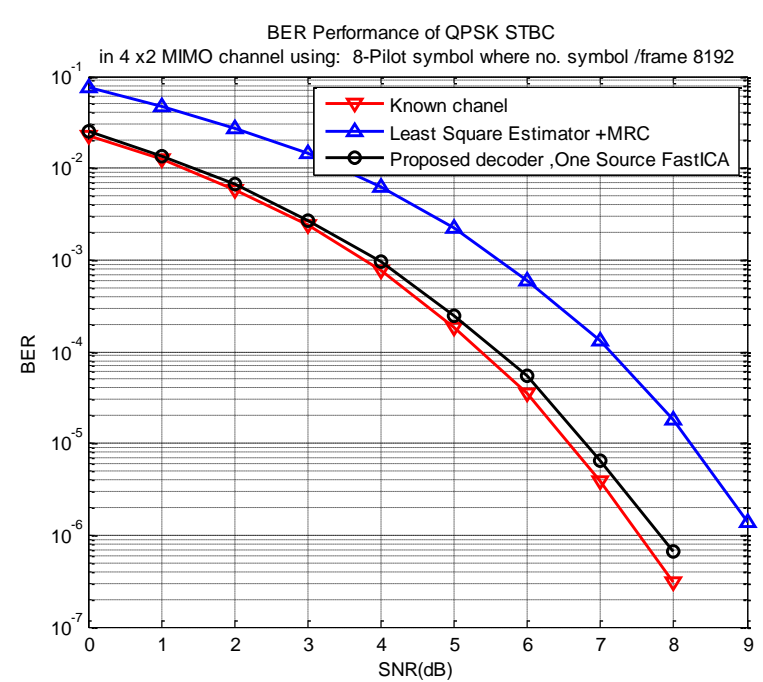

(a)

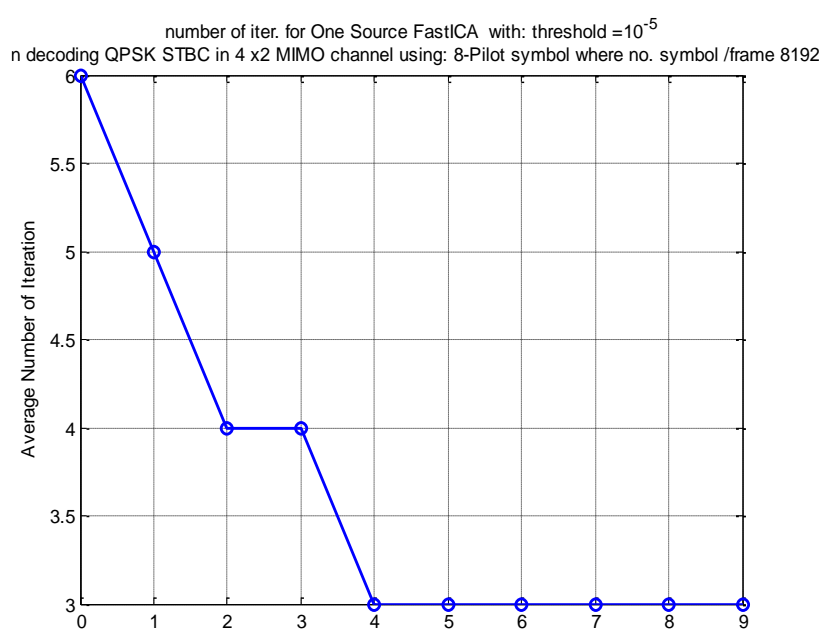

(b)

Figure. 8 : (a) Bit error rate and (b) the number of iterations for $4 \times 2$ MIMO STBC using $N_{s}=8192$

\section{Case 2: $N_{r}<N_{t}$}

Fig. 8 shows the bit error rate and the number of iterations in $4 \times 2$ MIMO STBC systems using 8 pilot symbols, where $N_{s}=8192$ symbols /frame is used. From the proposed decoder results, it is obvious that bit error rate analysis works efficiently although of that number of receiver antenna is less than a number of transmitter antennas. It can be also denoted that when $N_{r}<N_{t}$, the number of iterations for FastICA is increased.

\section{Case $3 N_{r}>N_{t}$}

Fig. 9 shows the bit error rate and the number of iterations in $4 \times 5$ MIMO STBC systems using 8 pilot symbols, where $N_{s}=8192$ symbols/ frame is used. From the proposed decoder results, it is obvious that the bit error rate analysis is better than conventional decoder for any number of receiver antennas.

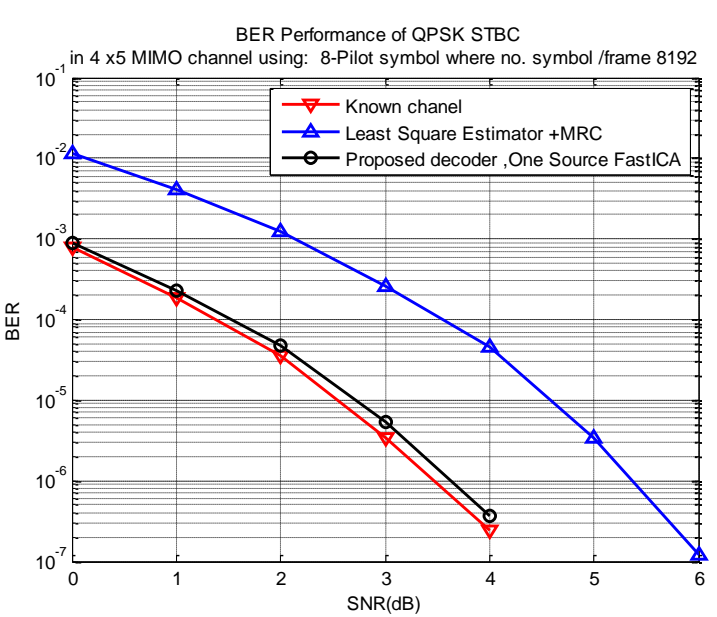

(a)

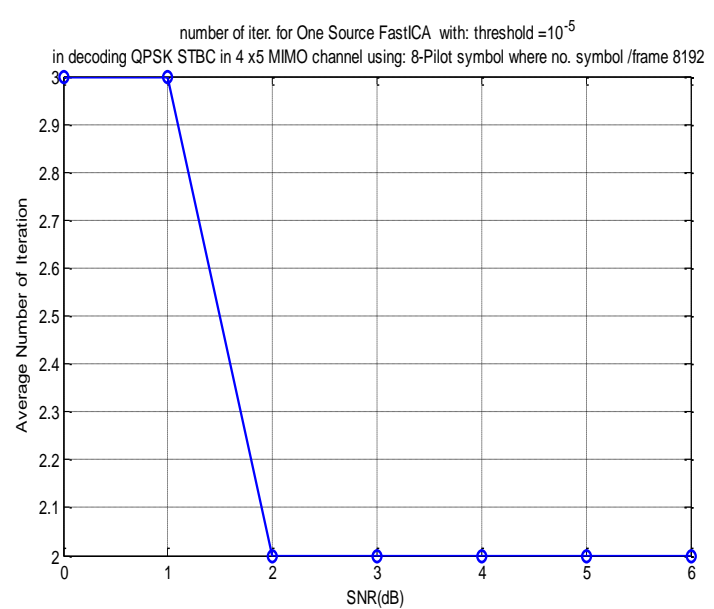

(b)

Figure. 9 Bit error rate and the number of iterations for $4 \times 5$ MIMO STBC using $N_{s}=8192$

\section{Comparing with other works}

Recently many researchers have been developed and discussed MIMO STBC such as [22] and [23], where [22] introduces minimum mutual information (MMI)- independent component analysis (ICA) algorithm has been used for extracting source signals and takes two transmitters ( $2 \times 2$ system) as a case study while [23] presents Kurtosis GPSO based BSS algorithm is applied to the decomposition model. The Fast-ICA system based on proposed mixing model has been performed in this paper which shows the complexity is very low, the speed is high and BER performance is excellent. The proposed design takes $4 \times 4$ system as a case study.

\section{Conclusion}

From Simulation results for classical decoder it denotes that, if the training symbols number is increased the performance of bit error rate of MIMO STBC is improved. At the same time, the transmission efficiencies have been reduced by this 
technique because the requirement of training symbols are combined with data symbols at the transmitter.

Based on statically analysis of mixing system shown in Fig. 5, it found that that optimum quadratic function for FastICA algorithm was $\log (\cosh (u))$ function.

Form Fig. 7 it can be denoted that BER (at SNR $=5$ ) improved from $10^{-6}$ into $4 \times 10^{-7}$ when number of symbols per frame increased from 2048 into 8192 . In general, it can be concluded that excellent BER performance for the new decoder associated with a high frame length.

During the simulation, it's found that performance of new decoder as compared with classical decoder at BER $=10^{-6}$, provided coding gain $=1 \mathrm{~dB}$ in case of $4 \times 2$ MIMO STBC system (Fig. 7 (C), and coding gain $=1.45 \mathrm{~dB}$ in case of $4 \times 4$ MIMO STBC system and coding gain $=1.76 \mathrm{~dB}$ in case of $4 \times 4$ MIMO STBC system. The important point that can be concluded that the new decoder is very fast where it needs about 2 4 iteration only. At the same time BER performance of this decoder is always butter than classical decoder. Therefore, it can be saying that the features of proposed decoder have an excellent bit error rate performance, low complexity and high speed that can work with any MIMO STBC using only fewer pilot symbols (8 symbols).

\section{References}

[1] S. M. Alamouti, "A Simple Transmit Diversity Technique for Wireless Communications", IEEE Journal on Select Areas in Communications, Vol. 16, No. 8, pp.1451-1458, October 1998.

[2] S. G. Glisic, Advance Wireless Communications, $2^{\text {nd }}$ Edition Johan Wiley \& Sons, Ltd, 2007.

[3] J. R. Hampton, Introduction to MIMO Communications, Cambridge University Press, 2014.

[4] G. Qian, L. Li and M. Luo, "On the Blind Channel Identifiability of MIMO-STBC Systems Using Noncircular Complex FastICA Algorithm", Circuits, Systems, and Signal Processing, Vol. 33, No. 6, pp 1859-1881, 2014.

[5] V. Tarokh, H. Jafarkhani, and A. R. Calderbank, "Space-Time Block Codes from Orthogonal Design", IEEE Transactions on Information Theory, Vol. 45, No. 5, pp. 1456-1467, 1999.

[6] V. Tarokh, H. Jafarkhani, and A. R. Calderbank, "Space-Time Block Coding for Wireless Communications: Performance Results", IEEE Journal on Selected Areas in Communications, Vol. 17, No. 3, pp. 451- 460, 1999.
[7] L. A. Perişoară, "BER Analysis of STBC Codes for MIMO Rayleigh Flat Fading Channels", Tel for Journal, Vol. 4, No. 2, pp. 78-82, 2012.

[8] S. D. Santumon and B. R. Sujatha, "Space-Time Block Coding (STBC) for Wireless Networks", International Journal of Distributed and Parallel Systems (IJDPS), Vol. 3, No. 4, pp. 183- 195, 2012.

[9] M. Biguesh and A. B. Gershman, "TrainingBased MIMO channel Estimation: a Study of Estimator Tradeoffs and Optimal Training Signals", IEEE Transactions on Signal Processing, Vol. 54, No. 3, pp. 884-893, 2006.

[10] C. Budianu and L. Tong, "Channel Estimation for Space-Time Orthogonal Block Codes", IEEE Transactions on Signal Processing, Vol. 50, No. 10, pp. 2515-2528, 2002.

[11] G. Taricco and E. Biglieri, "Space-Time Decoding with Imperfect Channel Estimation", IEEE Transactions on Wireless Communications, Vol. 4, No. 4, pp.1874-1888, 2005.

[12] A. Cichocki and S. I. Amari, "Adaptive Blind Signal and Image Processing: Learning Algorithms and Applications", Vol. 1, John Wiley \& Sons. 2002.

[13] V. Zarzoso, and P. Comon, "Robust Independent Component Analysis by Iterative Maximization of the Kurtosis Contrast with Algebraic Optimal Step Size", IEEE Transactions on Neural Networks, Vol. 21, No. 2, pp. 248-261, 2010.

[14] A. Hyvärinen and E. Oja, "Independent Component Analysis: Algorithms and Applications", Neural Networks, Vol. 13, No. 4, pp.1-31, 2000.

[15] A. Hyvärinen, "Survey on Independent Component Analysis", Neural Computing Surveys, Vol. 2, pp. 94-128, 1999.

[16] A. Hyvarinen, "Fast and Robust Fixed-Point Algorithms for Independent Component Analysis", IEEE Transactions on Neural Networks, Vol. 10, No. 3, pp.626- 634, 1999.

[17] Z. Koldovsky, P. Tichavsky, and E. Oja, "Efficient Variant of Algorithm FastICA for Independent Component Analysis Attaining the Camer - Rao Lower Bound", IEEE Transactions on Neural Networks, Vol. 17, No. 5, pp. 12651277, 2006.

[18] A. Hyvarinen, "Fast and Robust Fixed-Point Algorithms for Independent Component Analysis", IEEE Transactions on Neural Networks, Vol. 10, No. 3, pp. 626-634, 1999.

[19] S. Abdul-Kadhim, "Improve BER Performance of QPSK-Alamouti's STBC's Decoder Using Source Extraction Method Based on (R-Im) 
Decomposition Model", Al-Qadisiya Journal for Engineering Sciences, Vol. 7, No. 1, pp. 5667, 2014.

[20] T-H. Liu, "Comparisons of Two Real-Valued MIMO Signal Models and Their Associated ZFSIC Detectors over the Rayleigh Fading Channel", IEEE Transactions on Wireless Communications, Vol. 12, No. 12, pp. 60546066, 2013.

[21] S. Abdul-Kadhim and A. Q. J. Althahab, "A New DOA Estimation Approach for QPSK Alamouti STBC Using ICA Technique-Based PSO Algorithm", International Journal of Communication Systems Vol. 32, No. 2, pp. 3858-3870, 2019.

[22] N. S. Ali, K. K. Abdalla, and S. AbdulKadhim, "BER Performance Improvement of Alamouti MIMO-STBC Decoder Using Mutual Information Method", Journal of Physics: Conf. Series, Vol. 1530, 2020. doi:10.1088/17426596/1530/1/012016, 2020.

[23] S. A. K. Alrufaiaat, A. Q. J. Althahab, "Robust decoding strategy of MIMO-STBC using one source Kurtosis based GPSO algorithm", Journal of Ambient Intelligence and Humanized Computing, https://doi.org/10.1007/s12652020-02288-1, 2020. 